\title{
Macroscopic behavior of a reinforced elastomer: micromechanical modelling and validation
}

\author{
Vanessa Boucharta ${ }^{a}$, Mathias Brieu, Djimedo Kondo and Moussa Naït Abdelaziz \\ Laboratoire de Mécanique de Lille, Boulevard Paul Langevin, 59655 Villeneuve d'Ascq, France
}

Received 15 March 2007, Accepted 7 June 2007

\begin{abstract}
In the present study, we propose an evaluation of a non-linear homogenization model applied to hyperelastic composites having random microstructure. This modelling approach consists in a 3D implementation of the second order method introduced by Ponte Castañeda \& Tiberio [1]. We first recall the basic principles of this method. Then, we investigate a composite made up of an hyperelastic matrix reinforced by spherical deformable or rigid particles. Computational issues of the micromechanical model are discussed and some obtained results allow to demonstrate the reinforcement effect of the particles. In order to provide a rigorous evaluation of the methodology, finite elements computations, on an unit cell, are performed and compared to the predictions of the model. Finally, a confrontation with experimental results is provided.
\end{abstract}

Key words: Non-linear homogenization / hyperelasticity / second order method / elastomers / finite deformations

\begin{abstract}
Résumé - Comportement macroscopique d'un élastomère renforcé : modélisation micromécanique et validation. Dans cette étude, nous proposons l'évaluation d'un modèle d'homogénéisation non-linéaire appliqué aux composites hyperélastiques à microstructure aléatoire. Cette modélisation repose sur une mise en œuvre, dans un contexte 3D, de la méthode du second ordre introduite par Ponte Castañeda \& Tiberio [1]. Nous rappelons d'abord les principes de base de la méthode. Puis, nous étudions le cas d'un composite biphasé, constitué d'une matrice hyperélastique renforcée par des particules sphériques déformables ou rigides. L'implémentation numérique du modèle micromécanique est discutée et les résultats obtenus sont présentés afin de démontrer l'effet de renforcement des particules. Afin de fournir une évaluation rigoureuse de la démarche, des calculs par éléments-finis sur une cellule de base sont réalisés pour être comparés aux prédictions du modèle. Enfin, une confrontation aux données expérimentales est fournie.
\end{abstract}

Mots clés : Homogénéisation non-linéaire / hyperélasticité / méthode du second ordre / élastomères / grandes déformations

\section{Introduction}

Thanks to their remarkable properties of flexibility, deformability and resistance to high deformation levels, rubber-like materials are used in various industrial applications, in particular in automobile, spacial, aeronautic, railways or pneumatic industries. In order to improve their mechanical properties, elastomeric materials are commonly reinforced by particles or by fibers. A large number of publications attests the interest provided by the study of these reinforced materials.

\footnotetext{
${ }^{a}$ Corresponding author:

vanessa.bouchart@ed.univ-lille1.fr
}

Because of the reinforcements, one of the principal task is to characterize and model the macroscopic mechanical behavior of these complex materials. From the modelling point of view, two different approaches can be followed to predict the behavior of rubber-like reinforced materials under finite deformation. The first approach is phenomenological and provides simple models which can be calibrated by using very little experimental database. However, it is generally recognized that, especially for arbitrary loading paths, the predictive capabilities of these models remain very limited. A way to overcome these limitations consists to use micromechanics-based approaches which allow to link the macroscopic behavior of the material to its heterogeneous 


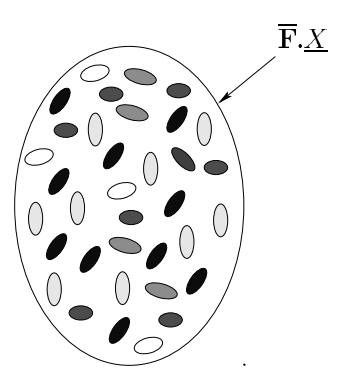

Fig. 1. Representative elementary volume (r.e.v.) of a reinforced material.

microstructure. In addition to homogenization models for periodic microstructures Brieu \& Devries [2], Lahellec et al. [3], there are various attempts in the literature devoted to materials with random microstructures. Within these ones, we focus on the recent developments of non-linear homogenization techniques proposed by PonteCastañeda [4]. In this framework, estimates are due to Ponte Castañeda \& Tiberio [1] (see also Lopez-Pamies $\&$ Ponte-Castañeda [5]). They have already been used for the modelling of fiber-reinforced materials. Thus, in order to extend the domain of applicability of this method, we propose an implementation and a validation of the nonlinear homogenization method for the case of an elastomer reinforced by deformable or rigid spherical particles. It is worthnoticing that the study is performed in a tridimensionnal context whereas it is bidimensionnal in the case of fiber-reinforced materials. The model is first evaluated by comparisons of its predictions to finite-elements computations on an unit cell. Finally, a confrontation with experimental results is provided.

\section{Homogenization of hyperelastic composites}

The heterogeneous and non-linear character of the studied materials has motivated the implementation and the use of a non-linear homogenization method for composites with random microstructure. The micromechanical modelling is based on the second order theory developed by Ponte Castañeda [4] and extended by Ponte Castañeda \& Tiberio [1] for hyperelastic behavior.

\subsection{Basics concepts}

Consider a representative elementary volume (r.e.v.) of hyperelastic reinforced materials, occupying a volume $V_{0}$ in the reference configuration. This composite is made up of particles embedded in a rubber-like matrix (see Fig. 1). The classical conditions of scale separation are supposed to be fulfilled and the composite is subjected to homogeneous strain conditions at the boundary of the r.e.v.: $\overline{\boldsymbol{F}}=\langle\boldsymbol{F}\rangle, \boldsymbol{F}$ being the deformation gradient tensor and $\langle$.$\rangle represents the volume average over V_{0}$.

The particles are randomly distributed in the r.e.v. and as well as the matrix, they are assumed to have hyperelastic constitutive behaviors which are a priori given and modelled by strain energy densities: $W^{(r)}(\boldsymbol{F})^{1}$, such that the local strain energy density function of the composite is given by:

$$
W(\underline{X}, \boldsymbol{F})=\sum_{r=1}^{N} \theta^{(r)}(\underline{X}) W^{(r)}(\boldsymbol{F})
$$

The quantity $\underline{X}$ represents the position of a material point $M_{0}$ in the reference configuration and $\theta^{(r)}(\underline{X})$ is the characteristic function of the phase $(r)$, equal to 1 if $\underline{X}$ is inside phase $(r)$ and zero otherwise.

A classical result, due to Hill [6], is that the overall behavior of the composite is obtained from the macroscopic strain energy density function $\widetilde{W}$. The macroscopic constitutive relation between the macroscopic stress (Piola Kirchoff 1) $\overline{\boldsymbol{T}}=\langle\boldsymbol{T}\rangle$ and the macroscopic deformation gradient $\overline{\boldsymbol{F}}=\langle\boldsymbol{F}\rangle$ is given by:

$$
\overline{\boldsymbol{T}}(\overline{\boldsymbol{F}})=\frac{\partial \widetilde{W}(\overline{\boldsymbol{F}})}{\partial \overline{\boldsymbol{F}}}
$$

\subsection{Macroscopic strain energy density determined with the second order method}

The second order homogenization procedure allows to estimate the strain energy density function of the hyperelastic composite from the behavior of its constituents. The method makes use of a general linearization based on a Taylor expansion of the local strain energy density around reference deformation gradients which are supposed to be uniform by phase and are found equal to $\overline{\boldsymbol{F}}^{(r)}$ (average of $\boldsymbol{F}$ on the phase $(r)$ in the linear comparison composite introduced by [4]). The final result coming from this method leads to the following expression of the macroscopic strain energy density $\widetilde{W}(\overline{\boldsymbol{F}})[1]$ :

$\widetilde{W}(\overline{\boldsymbol{F}})=$

$$
\sum_{r=1}^{N} c_{r}\left[W^{(r)}\left(\overline{\boldsymbol{F}}^{(r)}\right)+\frac{1}{2} \boldsymbol{T}^{(r)}\left(\overline{\boldsymbol{F}}^{(r)}\right):\left(\overline{\boldsymbol{F}}-\overline{\boldsymbol{F}}^{(r)}\right)\right]
$$

with $\boldsymbol{T}^{(r)}\left(\overline{\boldsymbol{F}}^{(r)}\right)=\frac{\partial W^{(r)}}{\partial \boldsymbol{F}}\left(\overline{\boldsymbol{F}}^{(r)}\right)$.

The corresponding macroscopic first Piola-Kirchoff stress tensor reads:

$$
\begin{aligned}
\overline{\boldsymbol{T}}(\overline{\boldsymbol{F}})=\sum_{r=1}^{N} \frac{c_{r}}{2}\left[\boldsymbol{T}^{(r)}\left(\overline{\boldsymbol{F}}^{(r)}\right)+\left[\boldsymbol{T}^{(r)}\left(\overline{\boldsymbol{F}}^{(r)}\right)+\right.\right. \\
\left.\left.\mathbb{L}^{(r)}\left(\overline{\boldsymbol{F}}^{(r)}\right):\left(\overline{\boldsymbol{F}}-\overline{\boldsymbol{F}}^{(r)}\right)\right]: \frac{\partial \overline{\boldsymbol{F}}^{(r)}}{\partial \overline{\boldsymbol{F}}}\right]
\end{aligned}
$$

where

$$
\mathbb{L}^{(r)}\left(\overline{\boldsymbol{F}}^{(r)}\right)=\mathbb{L}_{\mathrm{t}}^{(r)}\left(\overline{\boldsymbol{F}}^{(r)}\right)=\frac{\partial^{2} W^{(r)}}{\partial \boldsymbol{F}^{2}}\left(\overline{\boldsymbol{F}}^{(r)}\right)
$$

1 The superscript $(r)$ stands for a constituent $r$. 
It is emphasized that the only unknown in the above expressions are the average deformation gradient in each phase. These quantities, $\overline{\boldsymbol{F}}^{(r)}$ can be computed from the resolution of a thermoelastic problem related to the linear comparison composite.

\section{Application to an hyperelastic matrix reinforced with spherical particles}

\subsection{Thermoelasticity problem to be solved}

For a two-phase composite, the resolution of the thermoelasticity problem associated to the second order method and necessary required for the determination of $\overline{\boldsymbol{F}}^{(1)}$ and $\overline{\boldsymbol{F}}^{(2)}$, is performed thanks to the result provided by the theorem of Levin [7]:

$$
\begin{aligned}
\overline{\boldsymbol{F}}^{(r)} & =\mathbb{A}^{(r)}\left(\overline{\boldsymbol{F}}^{(r)}\right): \\
\overline{\boldsymbol{F}} & +\left(\mathbb{A}^{(r)}\left(\overline{\boldsymbol{F}}^{(r)}\right)-\mathbb{I}\right):(\Delta \mathbb{L})^{-1}:(\Delta \tau) ; r=1,2
\end{aligned}
$$

where $\mathbb{A}^{(r)}$ is the localization tensor associated to phase $(r)$ in the linear elasticity problem, $\triangle \mathbb{L}=\mathbb{L}^{(1)}\left(\overline{\boldsymbol{F}}^{(1)}\right)-$ $\mathbb{L}^{(2)}\left(\overline{\boldsymbol{F}}^{(2)}\right), \triangle \tau=\tau^{(1)}\left(\overline{\boldsymbol{F}}^{(1)}\right)-\tau^{(2)}\left(\overline{\boldsymbol{F}}^{(2)}\right)$ where $\tau^{(r)}=$ $\boldsymbol{T}^{(r)}\left(\overline{\boldsymbol{F}}^{(r)}\right)-\mathbb{L}^{r}: \overline{\boldsymbol{F}}^{(r)}$ is the polarization tensor. The localization tensors $\mathbb{A}^{(r)}$ depend on the chosen linear homogenization scheme. Moreover, for the two-phase material, one has $\widetilde{\mathbb{L}}=c^{(1)} \mathbb{L}^{(1)} \mathbb{A}^{(1)}+c^{(2)} \mathbb{L}^{(2)} \mathbb{A}^{(2)}$; this makes it possible to generate the corresponding estimates for $\widetilde{W}$.

In the present study, due to matrix-inclusion morphology of the studied material, we will consider two well known estimates: the Reuss scheme leading to $\widetilde{\mathbb{L}}^{-1}=$ $c^{(1)}\left(\mathbb{L}^{(1)}\right)^{-1}+c^{(2)}\left(\mathbb{L}^{(2)}\right)^{-1}$ and the Hashin-Shtrikman scheme $\left(\mathbb{L}^{(1)}\right.$ being taken as reference stiffness tensor) characterized by:

$$
\begin{aligned}
& \mathbb{A}^{(1)}=\left[c^{(1)} \mathbb{I}+c^{(2)}[\mathbb{I}-\mathbb{P}: \Delta \mathbb{L}]^{-1}\right]^{-1} ; \\
& \mathbb{A}^{(2)}=\left[\mathbb{I}-c^{(1)} \mathbb{P}: \Delta \mathbb{L}\right]^{-1}
\end{aligned}
$$

in which $\mathbb{P}$ is the Hill tensor.

The computation of the $\mathbb{P}$ tensor requires the determination of the anisotropic tensor $\mathbb{L}^{(1)}$. This one the later can be obtained by analytical computation from (2) while, because of the anisotropy of $\mathbb{L}^{(1)}, \mathbb{P}$ is determined by using a numerical integration over the surface of the unit sphere. Note that the spherical particles are assumed randomly dispersed, so that the composite remains statistically isotropic.

\subsection{Determination of the deformation gradients $\bar{F}^{(1)}$ and $\bar{F}^{(2)}$}

From a practical point of view for the calculation of $\overline{\boldsymbol{T}}(\overline{\boldsymbol{F}})$ by means of Equation (1), it is only necessary to determine $\overline{\boldsymbol{F}}^{(1)}$, the tensor $\overline{\boldsymbol{F}}^{(2)}$ being deduced from the classical average rule: $\overline{\boldsymbol{F}}=c^{(1)} \overline{\boldsymbol{F}}^{(1)}+c^{(2)} \overline{\boldsymbol{F}}^{(2)}$. The system of Equations (3) to be solved is a set of 9 non-linear equations with the 9 components of $\overline{\boldsymbol{F}}^{(1)}$ as unknowns. Thus, to solve this system, we use a Newton-Raphson method. The absence of a closed-form expression for $\mathbb{P}$ (in fact $\mathbb{P}^{-1}$ ), implies to perform a numerical derivation by finite difference to compute the $9 \times 9$ Jacobian matrix $\boldsymbol{J}$ associated to the system. At this step, in order to have a good accuracy, a Ridders-Richardson method was implemented (see [8]).

\section{Uniaxial tensile loading simulations}

For uniaxial tensile loading, the corresponding macroscopic deformation gradient associated with compressible materials is written in the form:

$\overline{\boldsymbol{F}}=\operatorname{Diag}(\lambda, \alpha, \alpha), \lambda$ increasing from 1.0. Due to the deformation-based control of the uniaxial tensile loading applied to the composite, for a given $\lambda$, one has to determine the value of $\alpha$ which fulfills the uniaxial tensile condition: $\overline{\boldsymbol{T}}=\operatorname{Diag}\left(T_{11}, 0,0\right)$.

The matrix and the particles being assumed compressible, the above conditions can be obtained only by using an iterative procedure. For this, we have considered a dichotomy algorithm which, after convergence, yields the suitable value of $\alpha$ for a given $\lambda$.

In the present application, the matrix " 1 " and the particles " 2 " being isotropic, the corresponding strain energy densities $W^{(1)}$ and $W^{(2)}$ can be classically expressed as function of the 3 invariants, $i_{1}, i_{2}$ and $i_{3}$ of the dilatation tensor $\boldsymbol{C}=\boldsymbol{F}^{t} . \boldsymbol{F}[9]: W(\underline{X}, \boldsymbol{F})=W\left(\underline{X}, i_{1}, i_{2}, i_{3}\right)$.

Since one of the objectives is to compare the model predictions with numerical (Finite-Element) computations on unit cell (see Sect. 5), we adopt for the homogenization method, strain energy densities available in the Finite-Element Software Abaqus. Thus, we consider for the matrix, the strain energy density associated with the Mooney-Rivlin hyperelastic material:

$$
W^{(1)}(\boldsymbol{F})=C_{10}\left(\overline{i_{1}}-3\right)+C_{01}\left(\overline{i_{2}}-3\right)+\frac{1}{D_{1}}(J-1)^{2}
$$

where $J=\operatorname{det}(\boldsymbol{F})=\sqrt{i_{3}}, \overline{i_{1}}=J^{-2 / 3} i_{1}, \overline{i_{2}}=J^{-4 / 3} i_{2}$. For the particles, we adopt a Neo Hookean model: $W^{(2)}(\boldsymbol{F})=C_{10}^{\prime}\left(\overline{i_{1}}-3\right)+\frac{1}{D_{1}^{\prime}}(J-1)^{2}$ which easily allows to account for the case of a rigid particle by taking a high value of $C_{10}^{\prime}$ and a small value of $D_{1}^{\prime}$. Indeed, in the limiting case of small strains, the material behavior can be seen as linear and isotropic one with initial bulk modulus $K^{\prime}=\frac{2}{D_{1}^{\prime}}$ and initial shear modulus $\mu^{\prime}=2 C_{10}^{\prime}$.

\subsection{Case of rigid particles}

The parameters used in the simulations are for the matrix: $C_{10}=C_{01}=1 \mathrm{MPa} ; D_{1}=2 / 3 \mathrm{MPa}^{-1}$. The 


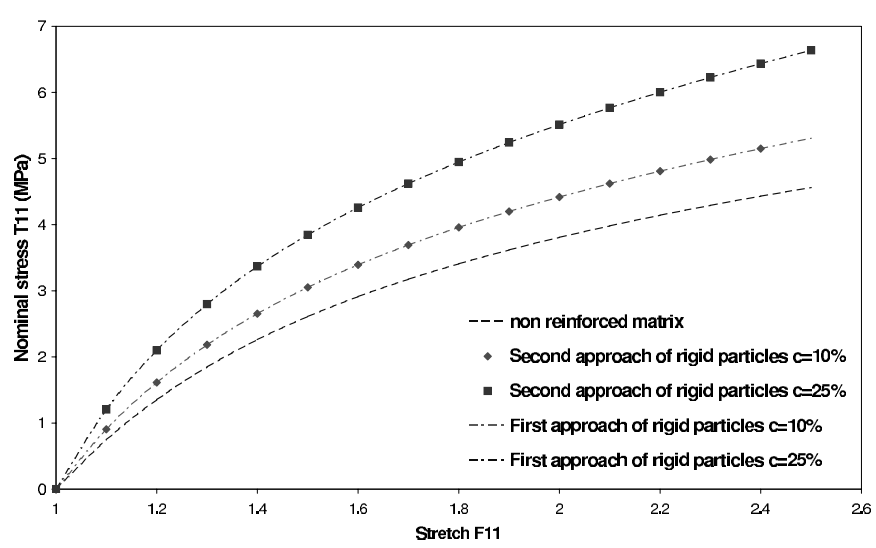

Fig. 2. Hashin-Shtrikman models predictions for the second order approaches for a matrix reinforced with rigid particles.

modelling of rigid particles can be done in two manners. First, they can be modelled by taking in the Neo-Hookean density a high value of $C_{10}^{\prime}$ and a small value for $D_{1}^{\prime}$. The second way, studied in Appendix A, consists to introduce directly in the second order method the assumption of rigid particles $\left(\boldsymbol{F}^{(2)} \approx \boldsymbol{I}\right)$ (see [1]). As already seen, the advantage of this second approach is to avoid the numerical resolution of the thermoelastic problem as required in the first approach. We aim here to compare these two approaches of modelling rigid particles. Figure 2 shows the overall response of the rigidly-reinforced material under uniaxial loading, as predicted by the Hashin-Shtrikman bound-based model respectively. The two approaches of modelling give the same predictions. Clearly enough, this provides a first validation of the general formulation of the micromechanical model which is based on the assumption of deformable particles.

\subsection{Case of deformable particles}

We use the following parameters for the particles: $C_{10}^{\prime}=5 \mathrm{MPa} ; D_{1}^{\prime}=0.8 \mathrm{MPa}^{-1}$. Figure 3 shows the macroscopic responses to an uniaxial tensile loading predicted by the second order homogenization method (nominal stress $T_{11}$ as function of $\overline{\boldsymbol{F}}_{11}$ ) for 2 volume fractions of particles (10\% and 25\%). From this figure, we note that the presence of the deformable particles has a clear effect on the macroscopic behavior of the material. A significant difference is observed between the predictions of the 2 considered homogenization schemes; the HashinShtrikman bounds-based model predicts, for a given level of uniaxial stress, a deformation less important than the one obtained with the Reuss model. The same observation is done in the case of rigid particles.

\section{Validation of the models by comparison with finite-elements simulations}

In order to validate the models based on the second order method, we present in this section the comparisons
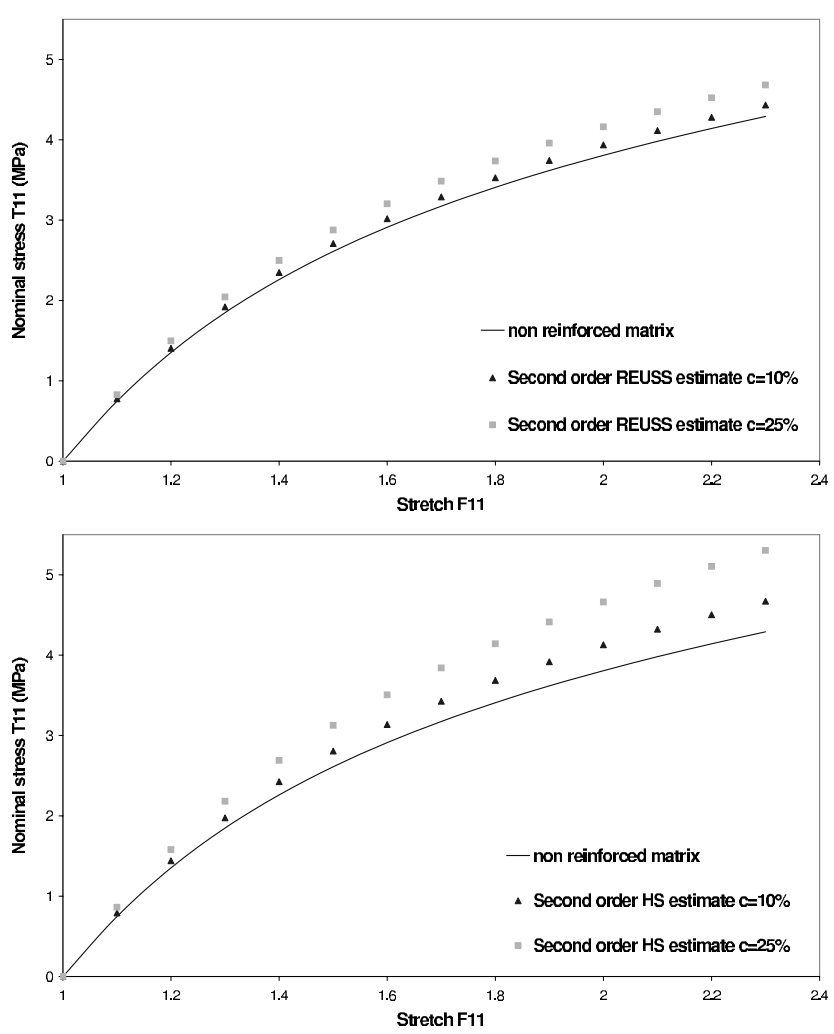

Fig. 3. Reuss and Hashin-Shtrikman models predictions in the case of deformable particles.

of their predictions with reference solutions obtained by finite-elements (FE) computations. For that, the same assumptions on the constitutive laws of constituents and on particles-matrix adherence are considered in the 2 computations (homogenization and finite-elements).

\subsection{Description of the finite-element simulations}

The reference solution FE is obtained by considering an unit cell described in Figure 4. This unit cell is already used by [10] in order to validate for elastoplastic materials the results of their homogenization code DIGIMAT. The plane $\left(\boldsymbol{e}_{\mathbf{1}}, \boldsymbol{e}_{\mathbf{2}}\right)$ being a symmetry plane of the problem, it is possible to only consider a quarter of section (see Fig. 4). The FE simulations have been performed in the software Abaqus. The boundary conditions considered to simulate an uniaxial tensile test are indicated in this figure. FE calculation makes it possible to evaluate the volume averages of the mechanical fields on the cell by a post-treatement.

\subsection{Comparison between the homogenization predictions and the FE calculations}

In the FE calculations, we model the matrix and the deformable particles with the same strain energy densities as previously indicated and with the same materials parameters. The predictions of the homogenization 


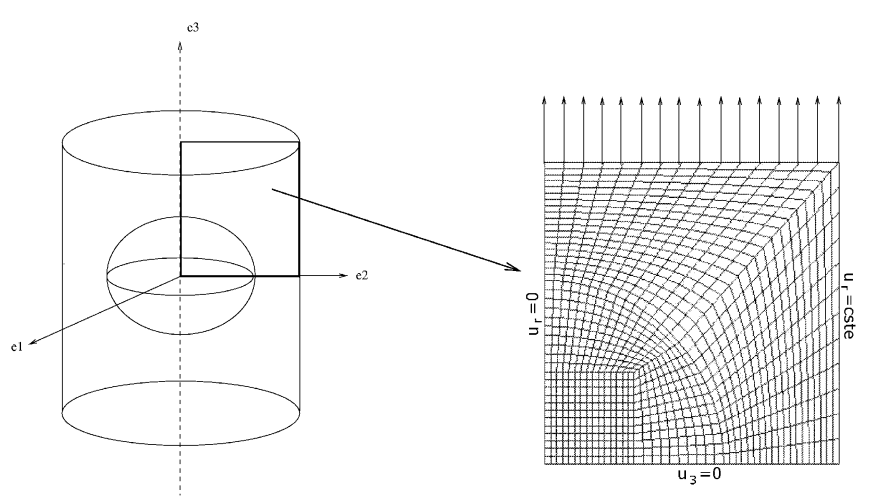

Fig. 4. Unit cell considered for the FE simulations.
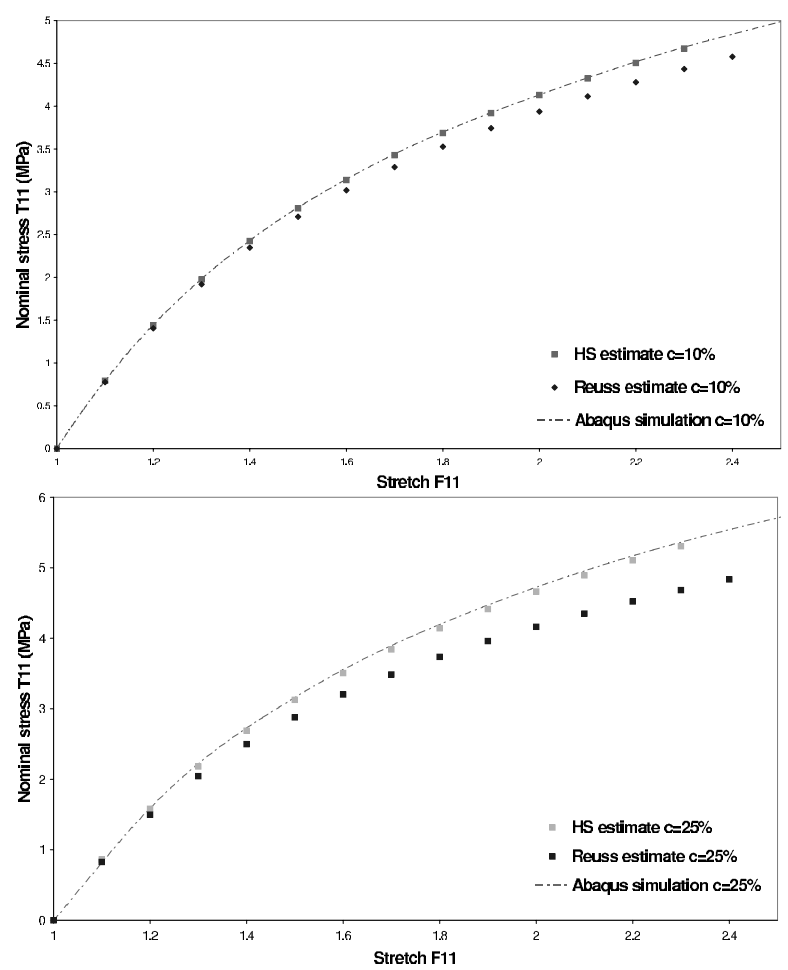

Fig. 5. Reuss and Hashin-Shtrikman predictions compared to the FE solutions for 2 volume fractions: $10 \%$ and $25 \%$.

method are compared in Figure 5 with the corresponding FE macroscopic results for 2 volume fractions of particles $(10 \%$ and $25 \%$ ). For each volume fraction, one observes that the Reuss model overestimates the macroscopic strain compared to FE results. This is undoubtedly due to the simplicity of the assumptions of this scheme. On the other hand we note a very good agreement between the Hashin-Strikman model (HS) and the numerical reference solution. This agreement illustrates the good capability of the model based on HS scheme to take into account the effect of the reinforcement and the nonlinearity of the composite through the second order method.

\section{Application to an EPDM matrix with spherical particles of PolyPropylene}

The homogenization approach being validated thanks to FE solutions, we now propose to compare its predictions (only HS model is considered) with experimental results for an elastomer (EPDM) reinforced by PolyPropylene particles $(\mathrm{PP})$.

In order to model the mechanical behavior of the reinforced material with the second-order homogenization method, it is necessary to use appropriate strain energy densities associated to each constituent. For this, we adopt for the matrix phase a strain energy density initially proposed by Lambert-Diani and Rey [11]:

$$
W^{(1)}=\int_{3}^{i_{1}} e^{\left(\alpha_{0}+\alpha_{1}\left(i_{1}-3\right)+\alpha_{2}\left(i_{1}-3\right)^{2}\right)} \mathrm{d} i_{1}+\int_{3}^{i_{2}} \beta_{1} i_{2}{ }^{\beta_{2}} \mathrm{~d} i_{2}
$$

and for the particles, we use a polynomial one:

$$
W^{(2)}=C_{1}\left(i_{1}-3\right)+C_{2}\left(i_{2}-3\right)-C_{3}\left(i_{3}-1\right)
$$

$\alpha_{0}, \alpha_{1}, \alpha_{2}, \beta_{1}, \beta_{2}, C_{1}, C_{2}$ and $C_{3}$ being parameters which have to be identified for the considered material.

\subsection{Experimental characterization and identification of the mechanical behaviors}

For the purpose of the identification of the local constitutive laws parameters, we perform uniaxial tensile tests on the elastomeric matrix and on the PolyPropylene.

\section{Experimental protocol}

The tensile tests were carried out on standardized samples of type $2[12]$, subjected to cyclic loadings with imposed maximum deformation. These tests, controlled in displacement, were carried out on a conventional machine (INSTRON 4302) equipped with a load cell of low capacity $(1 \mathrm{kN})$, compatible with the effort. The strain field on one face of the sample was mesured thanks to an optical extensometer. The deformation rate chosen for the whole study is $10^{-4} \mathrm{~s}^{-1}$, which makes it possible to ensure the quasi-static character of the tests. Note also that the tests were carried out several times in order to guarantee a good reproductibility.

The average responses of the matrix and of the PolyPropylene, obtained by means of these tests are given in Figures 6 and 7.

\section{Identification of the strain energy density}

The parameters can be calibrated by using the software Excell from experimental stress-strain data.

As in Section 4, the deformation gradient is written in the form $\boldsymbol{F}=\operatorname{Diag}(\lambda, \alpha, \alpha)$ and it is necessary to 


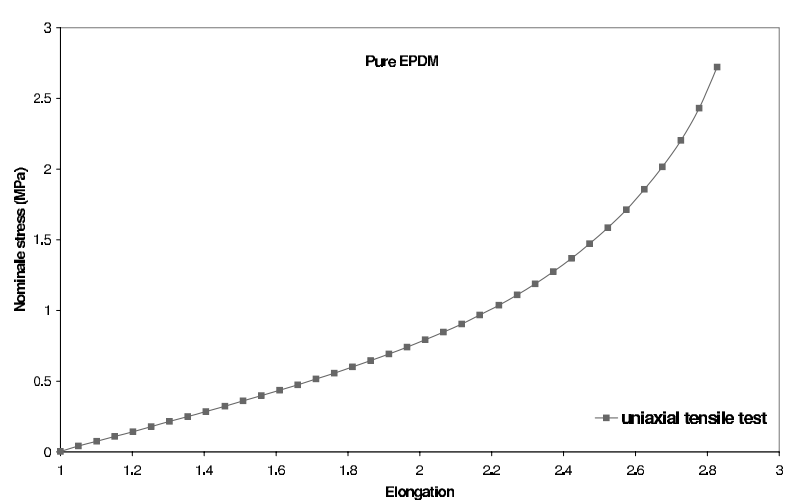

Fig. 6. Experimental mechanical response corresponding to the uniaxial tensile tests of the matrix.

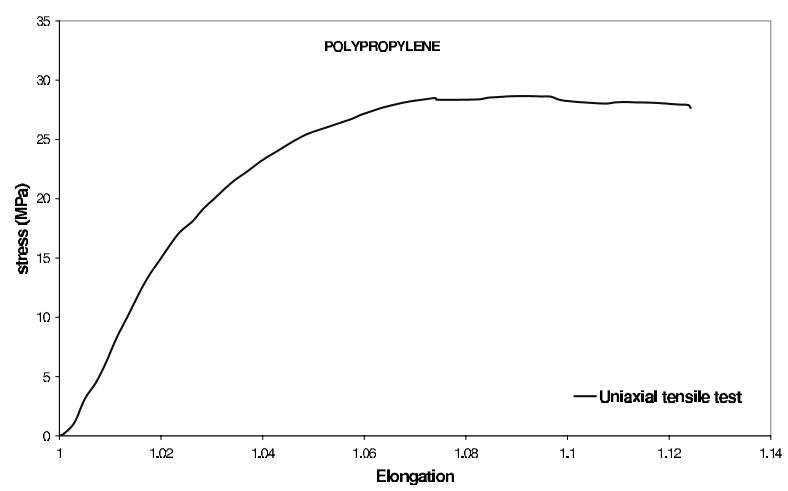

Fig. 7. Experimental mechanical response corresponding to the uniaxial tensile tests of the PolyPropylene.

determine $\alpha$ in order to fulfill the uniaxial tensile condition. Thus, the parameters are determined with the solver which minimizes the relative error in stress, $E$, while checking the uniaxial tensile condition.

For the $n$ stress/strain data pairs, the relative error measure $E$ is:

$$
E=\sum_{i=1}^{n}\left(1-\frac{\text { theoretical stress }}{\text { experimental stress }}\right)^{2}
$$

The theoretical stress is calculated by means of the strain-energy density adopted.

From the tests performed during this study, the following values were obtained:

$$
\begin{gathered}
e^{\alpha_{0}}=0.2246 \mathrm{MPa} ; \quad \alpha_{1}=0.013051 ; \quad \alpha_{2}=0.02400 ; \\
e^{\beta_{1}}=0.38104 \mathrm{MPa} ; \quad \beta_{2}=-2.032345 \\
C_{1}=-2250 \mathrm{MPa} ; \quad C_{2}=2437 \mathrm{MPa} ; \quad C_{3}=2624
\end{gathered}
$$

\subsection{Comparison between homogenization predictions and experimental data}

We compare the predictions of homogenization scheme of Hashin-Shtrikman with the experimental results (Fig. 8) for 2 volume fractions of particles 5\% and 10\%. In
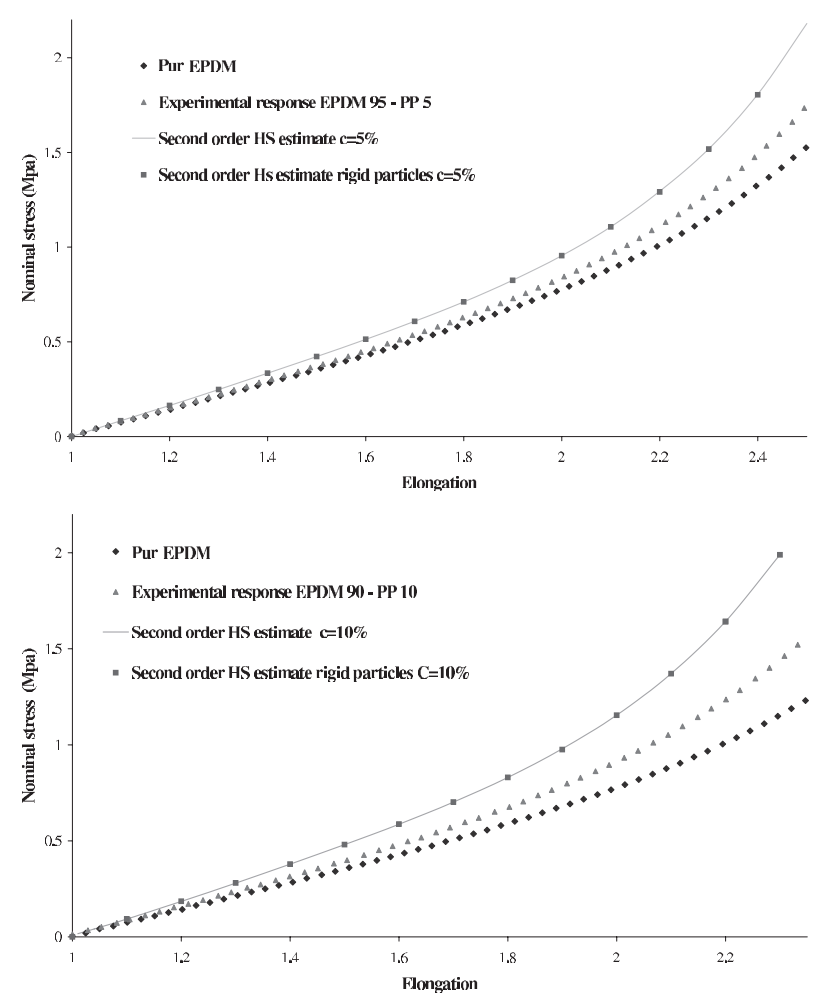

Fig. 8. Hashin-Shtrikman model predictions compared with the experimental results for a EPDM reinforced by $5 \%$ and $10 \%$ of particles.

this figure, we also report the homogenization predictions if the particles of $\mathrm{PP}$ are considered rigid with respect to the matrix i.e. we use the second order method as described in the Appendix A. We can see that the results of the 2 approaches to describe the particles behavior are quite similar. Consequently, it can be concluded that the assumption of rigid particles corresponds to the reality of the material. Moreover, one can see that, in spite of a good qualitative tendency of the predictions, the homogenization model overestimates the experimental stress for a given elongation.

\section{Conclusions}

The work presented in this study is devoted to the modelling of the mechanical behavior of hyperelastic materials reinforced by spherical particles. Micromechanical modelling, combining the second order method and the Hashin-Shtrikman homogenization scheme gives results in agreement with the reference solution obtained by FE calculations. The comparison of the homogenization scheme predictions with experimental data reveals a difference which can be due to the incapability of the current version of the micromechanical model to account for the damage phenomena which may occur during the deformation process. Indeed, the studied composite does not contain any compatibilizer i.e. the particles-matrix 
adherence is a priori not perfect. The incorporation of damage mechanism in the model is the subject of current researches.

\section{Appendix: Special case of rigid particles}

For various industrial applications, hyperelastic materials are reinforced by spherical particles (of volume fraction $c$ ) which can be approximatively considered as rigid ones with respect to the matrix behavior. Clearly enough, the particles "2" are embedded in an hyperelastic matrix "1" whose energy density function is denoted $W^{(1)}$. The assumption of rigid particles reads: $\overline{\boldsymbol{F}}^{(2)} \approx \boldsymbol{I}$. By the strain average rule, it follows that $\overline{\boldsymbol{F}}^{(1)}=\frac{1}{1-c}(\overline{\boldsymbol{F}}-c \boldsymbol{I})$.

Following [1] and since the thermoelasticity problem can be solved analytically, it is readily seen that the macroscopic stress tensor reads:

$$
\begin{aligned}
\overline{\boldsymbol{T}}(\overline{\boldsymbol{F}})=\boldsymbol{T}^{(1)}\left(\overline{\boldsymbol{F}}^{(1)}\right)+(\overline{\boldsymbol{F}}-\boldsymbol{I}):\left[\widetilde{\mathbb{L}}-\frac{1}{1-c} \mathbb{L}^{(1)}\right] \\
+\frac{1}{2}(\overline{\boldsymbol{F}}-\boldsymbol{I}): \frac{\partial}{\partial \overline{\boldsymbol{F}}}\left[\widetilde{\mathbb{L}}-\frac{1}{1-c} \mathbb{L}^{(1)}\right]:(\overline{\boldsymbol{F}}-\boldsymbol{I})
\end{aligned}
$$

Estimates of $\widetilde{\mathbb{L}}$ are obtained depends on the chosen linear homogenization schemes which generate the corresponding estimates for $\widetilde{W}$.

Note that in the case of rigid particles, the Reuss estimate leads to $\tilde{\mathbb{L}}=\frac{1}{1-c} \mathbb{L}^{(1)}$ and the Hashin-Shtrikman estimate, with $\mathbb{L}^{(1)}$ taken as reference modulus tensor, gives $\widetilde{\mathbb{L}}=\mathbb{L}^{(1)}+\frac{c}{1-c} \mathbb{P}^{-1}$.

As in the case of deformable particles, the computation of the Hill tensor has been done thanks to a numerical integration and its derivative with the RiddersRichardson method.

\section{References}

[1] P. Ponte Castañeda, E. Tiberio, A second order homogenization method in finite elasticity and applications to black-filled elastomers, J. Mech. Phys. Solids 48 (2000) 1389-1411

[2] M. Brieu, F. Devries, Homogénéisation de composites élastomères, Méthode et algorithme, Comptes Rendus de l'Académie des Sciences, Series IIB, Mechanics-PhysicsChemistry-Astronomy 326 (1998) 379-384

[3] N. Lahellec, F. Mazerolle, J.C. Michel, Second order estimate of the macroscopic behavior of periodic hyperelastic composites: theory and experimental validation, J. Mech. Phys. Solids 52 (2004) 27-49

[4] P. Ponte Castañeda, Exact second order estimates for the effective mechanical properties of non-linear composite materials, J. Mech. Phys. Solids 44 (1996) 827-862

[5] O. Lopez-Pamies, P. Ponte Castañeda, On the overall behavior, microstructure evolution, and macroscopic stability in reinforced rubbers at large deformations, IIApplication to cylindrical fibers, J. Mech. Phys. Solids 54 (2006) 831-863

[6] R. Hill, Convexity conditions and existence theorems in non-linear elasticity, Arch. Rat. Mech. Anal. 63 (1972) 337-403

[7] V.M. Levin, Thermek expansion coefficients of heterogeneous materials, Mekh. Tverd. Tela 2 (1967) 83-94

[8] W.H. Press, et al., Numerical recipes in Fortran, The art of scientific computing, 2nd Edition, Cambridge University Press, 1992

[9] R.W. Ogden, Non linear elastic deformations, Dover Publications Inc., New York, 1984

[10] I. Doghri, A. Ouaar, Homogenization of two-phase elastoplastic composite materials and structures study of tangent operators, cyclic plasticity and numerical algorithms, Inter. J. Solids and Structures 40 (2003) 16811712

[11] J. Lambert-Diani, C. Rey, New phenomenological behavior laws for rubbers and thermoplastics elastomers, Eur. J. Mech., A, Solids 18 (1999) 1027-1043

[12] R.P. Brown, Physical testing of Polymer, 3rd Edition, Chapman and Hall, 1984 